\title{
Changes in normal appearing spinal cord in multiple sclerosis: another brick in the wall
}

\author{
Alterações na medula espinal de aparência normal \\ na esclerose múltipla: mais um tijolo na parede
}

Leandro Tavares Lucato

\author{
Neuroradiologist, Instituto \\ de Radiologia do Hospital \\ das Clínicas da Faculdade de \\ Medicina da USP. CDB - Centro \\ de Diagnósticos Brasil, São Paulo \\ SP, Brazil. \\ Correspondence \\ Leandro Tavares Lucato \\ Rua Prof Pedreira de Freitas 372 / \\ apto 101 / bloco 2 \\ 03312-052 São Paulo SP - Brasil \\ E-mail: ltlucato@uol.com.br \\ Conflict of interest \\ There is no conflict of \\ interest to declare.
}

Received 30 June 2013

Accepted 08 July 2013
$\mathrm{I}$ $\mathrm{t}$ is needless to say how magnetic resonance (MR) has changed drastically the clinical management of multiple sclerosis (MS). Research in this field has also been much influenced by advances in MR techniques. density and FLAIR (fluid attenuated inversion recovery) images. Inflammatory activity can be observed by means of postcontrast T1-weighted images. Together, they are the basis of the present diagnostic criteria for dissemination in space and time that characterizes MS ${ }^{1}$.

However, the discrepancy between clinical manifestations of the disease and conventional MR metrics for lesion burden is well known, the so-called clinico-radiological paradox ${ }^{2}$. Some of the reasons listed to explain this dissociation include: inappropriate clinical rating, lack of histopathological specificity, neglecting spinal cord involvement, underestimating damage to the normal appearing brain tissue; and masking cortical adaptation effects².

MR has once again contributed enormously in this regard. Quantitative techniques (such as magnetization transfer and diffusion-weighted MR) disclose damage not only in conventional MR-visible lesions, but also in normal appearing tissues; MR spectroscopy adds metabolic information and functional MR demonstrates how cortical activation is affected during the course of the disease ${ }^{3}$.

A robust body of evidence has emerged from all these data, pointing out to our current understanding of MS as being not only a chronic inflammatory / demyelinating disease, but also a degenerative condition of the central nervous system. Another paradigm shift is related to the pathophysiology of MS: it is not only a white matter disease, characterized by focal inflammatory lesions, but it also involves more subtle and diffuse damage throughout white and gray matter ${ }^{3}$.

Specifically focusing on the spinal cord, it is a highly organized and clinically eloquent structure that is often affected by MS. Due to its peculiar anatomic arrangement, the cord is a useful model to assess the relations between inflammation, demyelination, and axonal pathology ${ }^{4}$.

Diffusion tensor imaging (DTI) is one of the most important quantitative techniques. It is a refinement of the conventional diffusion-weighted imaging, able to measure the random diffusional motion of water molecules and provide quantitative indices of structural and orientational features of tissues ${ }^{5,6}$. Among the most used DTI indices we can mention mean diffusivity (MD), which is a directionally averaged measure of the apparent diffusion coefficient, and fractional anisotropy (FA), which summarizes the orientational dependence of diffusivity?

More recently, new DTI metrics were introduced, probing new aspects of tissue structure and disease-related changes, such as axial $(\mathrm{AD})$ and radial diffusivity $(\mathrm{RD}) . \mathrm{AD}$ is considered to represent the water diffusion in parallel to the main structure of the white matter fibers, while $\mathrm{RD}$ represents the water diffusion perpendicular to them. It has been postulated, firstly in studies involving animals, that decreased $\mathrm{AD}$ and increased $\mathrm{RD}$ could represent surrogate markers of axonal damage and demyelination, respectively8.

Performing DTI of the spinal cord is much more challenging than of the brain, due to the small cross section of the cord, pulsatile cord motion, and field inhomogeneities caused by variations in susceptibility from nearby vertebrae ${ }^{9}$. Nevertheless, in spite of these limitations, 
the potential of DTI to detect spinal cord abnormalities in patients with MS has already been demonstrated. FA was reported to be associated with disability, not only in a single point in time ${ }^{7}$, but also in longitudinal studies ${ }^{10}$. Changes in $\mathrm{RD}$ seem to be an outcome predictor in MS patients with cervical cord relapse $e^{6,11}$. Another interesting point is that DTI changes in the spinal cord are not clearly correlated to the extent of brain and cord T2 lesion load in a longitudinal study of MS patients, which can support the idea that inflammatory demyelination (local and distant) may not be the only causative factor of intrinsic diffusivity cord changes, seen in patients with MS, leading to the possibility that neurodegeneration plays a role in this regard ${ }^{10}$.

In this issue of Arquivos de Neuro-Psiquiatria, Miraldi and colleagues add a new piece of information concerning this field of research, underscoring the importance of employing these techniques in a Brazilian cohort of MS patients. They focused on the analysis of normal-appearing cervical spinal cord, and although being unrelated to disability scores, DTI changes were found in MS patients compared to controls, including decreased FA (in the right column at the $\mathrm{C} 2$ level), and increased $\mathrm{MD}$ (in the right column at the $\mathrm{C} 7$ level) and $\mathrm{RD}$ (in the right column at the $\mathrm{C} 2$ level) ${ }^{12}$.

The asymmetry of their data is peculiar, with significant changes found only in the right column. However, recent published data showed that DTI changes are asymmetric in the lateral funiculi of the cervical spinal cord, and they correlated with asymmetric electrophysiological deficits for both arms and legs, possibly reflecting some structure-function relationship in the human spinal cord ${ }^{13}$.

Future studies, using new developments in MR sequences exploring the non-Gaussian properties of water diffusion in the spinal cord tissue, such as diffusion kurtosis ${ }^{14}$, might add new bricks to the wall that represents the advancement of knowledge about MS, such as the one added in the present issue of Arquivos de Neuro-Psiquiatria. However, it is important to underscore, as we are dealing with metaphorical bricks, the fact that we have to put them together meaningfully; otherwise, we do indeed just have a pile of bricks instead of a wall ${ }^{15}$.

\section{References}

1. Polman $\mathrm{CH}$, Reingold SC, Banwell B, et al. Diagnostic criteria for multiple sclerosis: 2010 revisions to the McDonald criteria. Ann Neurol 2011;69:292-302.

2. Barkhof F. The clinico-radiological paradox in multiple sclerosis revisited. Curr Opin Neurol 2002;15:239-245.

3. Filippi M, Rocca MA. MRI evidence for multiple sclerosis as a diffuse disease of the central nervous system. J Neurol 2005;252:S16-S24.

4. Filippi M, Rocca MA, Barkhof F, et al. Association between pathological and MRI findings in multiple sclerosis. Lancet Neurol 2012;11:349-360.

5. Pierpaoli C, Basser PJ. Toward a quantitative assessment of diffusion anisotropy. Magn Reson Med 1996;36:893-906.

6. Théaudin M, Saliou G, Ducot B, et al. Short-term evolution of spinal cord damage in multiple sclerosis: a diffusion tensor MRI study. Neuroradiology 2012;54:1171-1178.

7. Valsasina P, Rocca MA, Agosta F, et al. Mean diffusivity and fractional anisotropy histogram analysis of the cervical cord in MS patients. Neuroimage 2005;26:822-828.

8. Kim JH, Budde MD, Liang HF, et al. Detecting axon damage in spinal cord from a mouse model of multiple sclerosis. Neurobiol Dis 2006;21:626-632.
9. Bosma R, Stroman PW. Diffusion tensor imaging in the human spinal cord: development, limitations, and clinical applications. Crit Rev Biomed Eng 2012;40:1-20.

10. Agosta F, Absinta M, Sormani MP, et al. In vivo assessment of cervical cord damage in MS patients: a longitudinal diffusion tensor MRI study. Brain 2007;130:2211-2219.

11. Freund P, Wheeler-Kingshott C, Jackson J, Miller D, Thompson A, Ciccarelli $O$. Recovery after spinal cord relapse in multiple sclerosis is predicted by radial diffusivity. Mult Scler 2010;16:1193-1202.

12. Miraldi F, Lopes FCR, Costa JVA, Alves-Leon SV, Gasparetto EL. Diffusion tensor magnetic resonance imaging may show abnormalities in the normal-appearing cervical spinal cord from patients with multiple sclerosis. Arq Neuropsiquiatr 2013:71:580-583.

13. von Meyenburg J, Wilm BJ, Weck A, et al. Spinal cord diffusion-tensor imaging and motor-evoked potentials in multiple sclerosis patients: microstructural and functional asymmetry. Radiology 2013;267:869-879.

14. Raz E, Bester M, Sigmund EE, et al. A Better Characterization of Spinal Cord Damage in Multiple Sclerosis: A Diffusional Kurtosis Imaging Study. AJNR Am J Neuroradiol 2013:Epub ahead of print.

15. Altman DG. Building a metaphor: another brick in the wall? BMJ 2012;345:e8302. 